\title{
CUTTING FAMILIES OF CONVEX SETS
}

\author{
MEIR KATCHALSKI AND TED LEWIS
}

\begin{abstract}
A family of convex sets in the plane admits a common transversal if there is a straight line which intersects (cuts) each member of the family.

It is shown that there is a positive integer $k$ such that for any compact convex set $C$ in the plane and for any finite family $\mathscr{Q}$ of pairwise disjoint translates of $C$ : If each 3-membered subfamily of $\mathscr{Q}$ admits a common transversal then there is a subfamily $\mathscr{B}$ of $\mathscr{Q}$ such that $\mathscr{B}$ admits a common transversal and $|\mathfrak{Q} \backslash \mathscr{B}|<k$.
\end{abstract}

1. Introduction. A family $\mathscr{F}$ of subsets of the Euclidean plane is said to have property $T$, or to admit a common transversal, if there is a straight line which intersects every member of $\mathscr{F}$. The family has property $T(m), m$ a positive integer, if every $m$-membered subfamily of $\mathscr{F}$ has property $T$. Unless otherwise mentioned, we will assume throughout that $\mathscr{F}$ is a finite family of compact convex sets.

Many of the known results about common transversals (cf. [1, Chapter 5] and [8] for some of these facts) are concerned with the problem of finding conditions on $\mathscr{F}$ such that $T(m)$ implies $T$.

For obvious reasons the smallest value of $m$ for which this problem can be solved is $m=3$, and there are nontrivial families $\mathscr{F}$ for which $T(3)$ implies $T$; eg., if $\mathscr{F}$ consists of parallel straight line segments [11], [12], [13], or if $\mathscr{F}$ consists of infinitely many pairwise disjoint congruent compact sets with nonempty interiors [6]. On the other hand, there are also families for which $T(3)$ does not imply $T$. In particular, if $\mathscr{F}$ consists of $N$ pairwise disjoint translates of a parallelogram, then $T(5)$ implies $T$ and even $T(4)$ does not [4]. In this case, however, $T(4)$ does imply that there is a straight line intersecting $N-2$ members of $\mathscr{F}$ [9], and one is naturally led to ask if a similar conclusion can be drawn if it is assumed only that $\mathcal{F}$ has $T(3)$. In this note we show that such a conclusion is valid even without the assumption that the members of $\mathscr{F}$ are parallelograms.

We will say that a family $\mathcal{F}$ of subsets of the plane has property $T-k, k$ a nonnegative integer, if there is a straight line intersecting all but at most $k$ members of $\mathcal{F}$. Using this terminology, our main result is:

1. THEOREM. There exists a positive integer $k$ such that for any family $\mathscr{F}$ of pairwise disjoint translates of a compact convex set $C, T(3)$ implies $T-k$.

Theorem 1 will follow from the more general

Received by the editors April 2, 1979.

AMS (MOS) subject classifications (1970). Primary 52A35.

Key words and phrases. Common transversal, convex sets. 
2. THEOREM. Let $\mathcal{F}$ be any family of compact, convex subsets in the plane, and suppose that each member of $\mathcal{F}$ has diameter no greater than $r>0$. If $\mathcal{F}$ has $T(3)$, there are 3 discs of radius $3 r$ such that there is a common transversal for all members of $\mathcal{F}$ which do not intersect any of the discs.

A simple corollary of Theorem 2 which will be of use is

3. Corollary. Given $r>0, \beta>0$ and $n>0$, there is a positive integer $l=$ $l(r, \beta, n)$ such that $T(3)$ implies $T-l$ for any family $\mathcal{F}$ of compact convex sets satisfying the following conditions:

(i) each member of $\mathcal{F}$ has diameter no greater than $r$;

(ii) for every $n$-membered subfamily $\left\{A_{1}, \ldots, A_{n}\right\}$ of $\mathscr{F}$, the area of $\cup_{i=1}^{n} A_{i}$ is no smaller than $\beta \cdot n$.

The integer $k$ in Theorem 1 is universal for families of pairwise disjoint translates of a compact convex set. It is interesting to note that, for such families, it is unknown whether there is also a universal $m$ such that $T(m)$ implies $T$. (It is a long standing conjecture of Grünbaum [1], [4] that $m=5$ is universal.) In this regard, we also mention that for families $\mathscr{F}$ of arbitrary compact convex sets, there is no universal $m$ [8], not even when $\mathscr{F}$ consists of pairwise disjoint congruent rectangles [10].

2. Proofs. We require the following somewhat technical lemma.

4. LEMMA. Let $\left\{A_{1}, B, A_{2}\right\}$ be a family of three rectangles satisfying the following conditions:

(i) the edges are of length no greater than $r$ and parallel to the coordinate axes;

(ii) the distance between each two rectangles of the family is greater then $r$;

(iii) the horizontal coordinate axis $h$ intersects the three rectangles in the order $A_{1}, B, A_{2}$, and separates $A_{1} \cup A_{2}$ from $B$. (See Figure 1.)

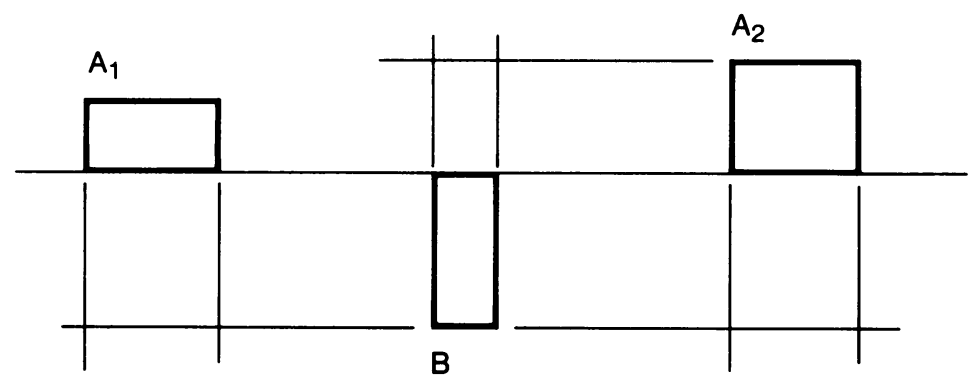

Figure 1

If $D$ is any rectangle satisfying (i) and if the family $\left\{A_{1}, B, A_{2}, D\right\}$ satisfies (ii) and also has property $T(3)$, then $D$ intersects the horizontal axis. (By the distance between two subsets $X$ and $Y$ mean $\inf \{\operatorname{dist}(x, y): x \in X, y \in Y\}$.)

Proof. We will show that $D$ can be neither strictly above $h$ nor strictly below $h$. Referring to Figure 1 , we note that if $D$ lies above $h$, then it must intersect the 
vertical strip defined by the extension of the vertical edges of $B$. (If, for example it were to the left, then $\left\{A_{2}, B, D\right\}$ would fail $T$.) Then, in order that $\left\{A_{1}, A_{2}, D\right\}$ have property $T, D$ cannot lie above the straight line through the uppermost horizontal edge of $A_{1} \cup A_{2}$. But then $\operatorname{dist}(D, B)<r$, a contradiction. On the other hand, again referring to Figure 1 , if $D$ lies strictly below $h$ then it must intersect one of two vertical half strips, the one below $A_{1}$, the other below $A_{2}$. (If $D$ lies between these strips, then $\left\{A_{1}, A_{2}, D\right\}$ fails $T$; if $D$ lies to the right of the strip below $A_{2}$, then $\left\{B, A_{2}, D\right\}$ fails $T$.) Assuming then that $D$ intersects the strip below $A_{1}$, we note that $D$ cannot lie strictly below the straight line containing the lower horizontal edge of $B$, for this would imply that $\left\{A_{1}, B, D\right\}$ fails $T$. But then, the distance between $A_{1}$ and $D$ is no greater than $r$, a contradiction. This completes the lemma.

Proof of Theorem 2. Letting $\mathscr{F}=\{C(\gamma): \gamma \in \Gamma\}$ be the family, choose $x(\gamma) \in$ $C(\gamma)$ for each $\gamma \in \Gamma$, and contract each $C(\gamma)$ about the point $x(\gamma)$ by a factor of $\lambda \in[0,1]$. Let $\lambda_{0}$ be the minimum value of $\lambda$ such that, for the contracted family $\mathcal{G}=\left\{C^{\prime}(\gamma): \gamma \in \Gamma\right\}$, there is a common transversal for every three members $C^{\prime}\left(\gamma_{1}\right), C^{\prime}\left(\gamma_{2}\right), C^{\prime}\left(\gamma_{3}\right)$ whenever $\operatorname{dist}\left(C\left(\gamma_{i}\right), C\left(\gamma_{j}\right)\right)>(1+\sqrt{2}) r, 1<i \neq j<3$. (If some three members of $\mathcal{G}$ are mutually separated by distances greater than $(1+\sqrt{2}) r$, then the existence of $\lambda_{0}$ can be established by standard compactness arguments. On the other hand if no three members of $\mathcal{G}$ are mutually separated by distances greater than $(1+\sqrt{2}) r$, then the theorem is trivially satisfied for the following reasons: either there is some pair $\left\{C^{\prime}\left(\gamma_{1}\right), C^{\prime}\left(\gamma_{2}\right)\right\}$ whose members are separated by a distance greater than $(1+\sqrt{2}) r$, or there is no such pair. In the latter case, let $\left\{C^{\prime}\left(\gamma_{1}\right), C^{\prime}\left(\gamma_{2}\right)\right\}$ be any pair chosen from $\mathcal{G}$. Each $C^{\prime}\left(\gamma_{i}\right)$ has diameter no greater than $r$, so by Jung's theorem (cf. [1, p. 112]) is contained in a disc of radius at most $r / \sqrt{3}$. Every other member of $\mathcal{G}$ must be with a distance $(1+\sqrt{2}) r$ from at least one of these two discs, and therefore every member of $\mathcal{G}$ intersects at least one of two discs of radius $(1+\sqrt{2}+1 / \sqrt{3}) r<3 r$. $)$

By the minimality of $\lambda_{0}$, there are three members of $\mathcal{G}$, say $C^{\prime}\left(\gamma_{1}\right), C^{\prime}\left(\gamma_{2}\right), C^{\prime}\left(\gamma_{3}\right)$ and a straight line $h$ intersecting the three sets in the order listed with $h$ separating $C^{\prime}\left(\gamma_{1}\right) \cup C^{\prime}\left(\gamma_{3}\right)$ from $C^{\prime}\left(\gamma_{2}\right)$. (See Figure 2.) Let $R_{i}$ be the smallest rectangle, with one pair of edges parallel to $h$, which contains $C^{\prime}\left(\gamma_{i}\right), i=1,2,3$, and set $R_{1}=A_{1}$, $R_{2}=B, R_{3}=A_{2}$.

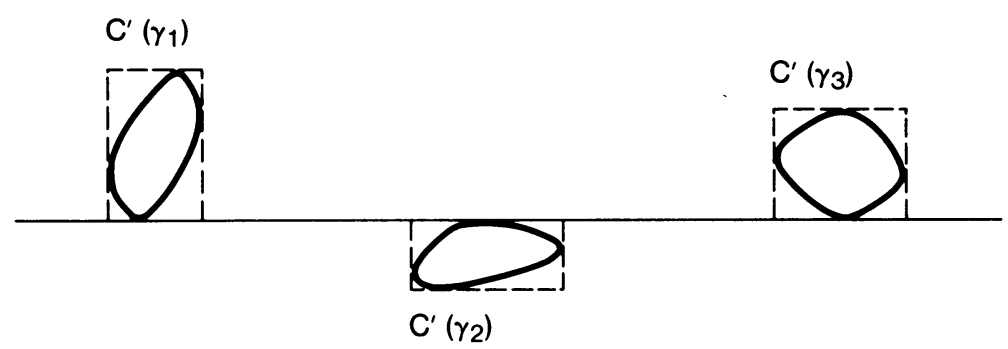

FIGURE 2

Assuming, without loss of generality, that $h$ is the horizontal axis and that $B$ lies below $h$, we claim that $A_{1}, B$, and $A_{2}$ satisfy all of the hypotheses of Lemma 4 . 
Since the diameter of $C^{\prime}\left(\gamma_{i}\right)$ is no greater than $r$, it is clear that (i) is satisfied, and (ii) is a consequence of the fact that $\operatorname{dist}\left(C^{\prime}\left(\gamma_{i}\right), C^{\prime}\left(\gamma_{j}\right)\right)>(1+\sqrt{2}) r$.

Now suppose that $C^{\prime}(\gamma)$ is any member of $\mathcal{G}$ such that $\operatorname{dist}\left(C^{\prime}(\gamma), C^{\prime}\left(\gamma_{i}\right)\right)>(1+\sqrt{2}) r$ for $i=1,2,3$. (If there is no such $C^{\prime}(\gamma)$, then every member of $\mathcal{G}$ intersects at least one of three fixed discs of radii $3 r$, each of these discs containing one of $C^{\prime}\left(\gamma_{i}\right), i=1,2,3$.) Let $D$ be the smallest rectangle with edges parallel to $h$ which contains $C^{\prime}(\gamma)$. Then, as above, $\operatorname{dist}\left(D, A_{1}\right)>r$, $\operatorname{dist}\left(D, A_{2}\right)>r, \operatorname{dist}(D, B)>r$, and the family $\left\{A_{1}, B, A_{2}, D\right\}$ has $T(3)$ since $\left\{C^{\prime}\left(\gamma_{1}\right), C^{\prime}\left(\gamma_{2}\right), C^{\prime}\left(\gamma_{3}\right), C^{\prime}(\gamma)\right\}$ does. Then, $D$, and therefore $C^{\prime}(\gamma)$, intersects $h$, completing the proof of Theorem 2 .

The corollary now follows from Theorem 2: condition (ii) of the corollary implies that, with the possible exception of $(n-1)$ members of $\mathcal{F}$, every member has area at least $\beta$. Thus three fixed discs of radius $4 r$ can contain at most $48 \pi r^{2} \beta^{-1}+(n-1)$ members of $\mathcal{F}$. From Theorem 2 , we have $l(r, \beta, n)=k \leqslant$ $48 \pi r^{2} \beta^{-1}+(n-1)$.

Proof of Theorem 1. For families of parallel segments, $T(3)$ implies $T$, so we may assume that $C$ has nonempty interior. In other words, each member of $\mathscr{F}$ has diameter $r$ and every 1 -membered subfamily of $\mathcal{F}$ has area $\beta$, and Theorem 1 will be an immediate consequence of the corollary once it is shown that we can always arrange things so that $r=\sqrt{2}$ and $\beta=\frac{1}{2}$. To do this, first enclose $C$ in a parallelogram $A$ in such a manner that the area of $C$ is at least one-half the area of $A$ (cf. Lemma 2 of [2]). Now let $\mathcal{L}$ be a linear transformation mapping $A$ into a square $S$ whose edges have length 1 . Then $\mathcal{L}(C)$ has area at least $\frac{1}{2}$ and diameter at most $\sqrt{2}$. Thus, $\mathcal{L}(\mathscr{F})$ (and hence $\mathscr{F}$ ) has property $T-k$ where $k=l\left(\sqrt{2}, \frac{1}{2}, 1\right)$, completing the proof of Theorem 1.

REMARKS. (i) Using $l(r, \beta, n)=l\left(\sqrt{2}, \frac{1}{2}, 1\right)$ provides an upper bound of $192 \pi$ for the value of $k$ in Theorem 1, and it seems clear that there is room for improvement. This seems much too large, and we in fact conjecture that the value $k=2$ is universal for families of disjoint translates of a compact convex set. However, even when the members of $\mathscr{F}$ are squares, or circles, it is not known whether $k=2$.

(ii) It is readily seen that disjointedness is necessary in Theorem 1.

(iii) Using a construction similar to that in [10], an example using congruent straight line segments will show that the value of $k$ in Theorem 1 does not hold if we allow rotations as well as translations. In other words, there is no universal $k$ for families consisting of pairwise disjoint congruent convex sets.

\section{REFERENCES}

1. L. Danzer, B. Grünbaum and V. Klee, Helly's theorem and its relatives, Convexity, Proc. Sympos. Pure Math., vol. 7, Amer. Math. Soc., Providence, R. I., 1963, pp. 101-180.

2. Th. Estermann, Über den Vektorenbereich eines Konvexen Körpers, Math. Z. 28 (1928), 471-475.

3. B. Grübaum, On a theorem of L. A. Santaló, Pacific J. Math. 5 (1955), 351-359.

4. On common transversals, Arch. Math. 9 (1958), 465-469.

5. Common transversals for families of sets, J. London Math. Soc. 35 (1960), 408-416.

6. H. Hadwiger, Über einen Satz Hellyscher Art, Arch. Math. 7 (1956), 377-379.

7. __ Uber Eibereiche mit gemeinsamer Treffgeraden, Portugal. Math. 16 (1957), 23-29. 
8. H. Hadwiger, H. Debrunner and V. Klee, Combinatorial geometry in the plane, Holt, Rinehart and Winston, New York, 1964.

9. M. Katchalski and T. Lewis, Cutting rectangles in the plane (to appear).

10. T. Lewis, Two counterexamples concerning transversals for convex subsets of the plane, Geometriae Dedicata (to appear).

11. L. Santaló, $A$ theorem on sets of parallelepipeds with parallel edges, Publ. Inst. Mat. Univ. Nac. Litoral (Rosario) 2 (1940), 49-60. (Spanish)

12. Supplement to the note: $A$ theorem on sets of parallelepipeds with parallel edges, Publ. Inst. Mat. Univ. Nac. Litoral (Rosario) 3 (1942), 202-210. (Spanish)

13. H. Rademacher and I. J. Schoenberg, Helly's theorem on convex domains and Tchebycheff's approximation problem, Canad. J. Math. 2 (1950), 245-256.

Faculty of Mathematics, Technion, Haifa, IsRael

Department of Mathematics, University of Alberta, Edmonton, Alberta T6G 2G1, Canada 